\title{
Gemcitabine fixed-dose rate infusion for the treatment of pancreatic carcinoma: a meta-analysis of randomized controlled trials
}

Jiqing $\mathrm{Xie}^{1 \dagger}$, Jing Yuan ${ }^{2+}$ and Laichun $\mathrm{Lu}^{3^{*}}$

\begin{abstract}
Background: Pre-clinical evidence shows that fixed dose rate (FDR) infusion of gemcitabine could optimize plasma concentration of gemcitabine, while the clinical efficacy and toxicity of FDR infusion of gemcitabine in advanced pancreatic carcinoma has not been systematically investigated. Thus, this meta-analysis was designed to ascertain this issue.
\end{abstract}

Methods: Databases of EMBASE, PubMed, and Cochrane Library were searched for eligible randomized controlled trials (RCTs). RCTs comparing FDR and standard 30-min infusion of gemcitabine in advanced pancreatic carcinoma were included. The primary endpoints were treatment efficacy (overall response rate, 1-year survival rate, median survival, and time to treatment failure) and toxicities were secondary endpoints (neutropenia, thrombocytopenia, anemia, and vomiting). Relative risks or mean differences and corresponding 95\% confidence intervals (Cls) were calculated.

Result: After careful and rigorous selection, 3 eligible RCTs including 764 patients of advanced pancreatic adenocarcinoma were included in this meta-analysis. For treatment efficacy, FDR gemcitabine provided significantly longer median survival over standard gemcitabine (Mean Difference $=1.24$ months, 95\% Cl: 0.39-2.09), while there was no statistical difference in other endpoints of treatment efficacy. For toxicities, patients with FDR gemcitabine experienced significantly more grade 3/4 hematological toxicities than those received standard gemcitabine (neutropenia, thrombocytopenia, and anemia), while there was no difference for vomiting.

Conclusion: Compared with standard 30-min infusion, FDR gemcitabine provide longer median survival, but increased the risk of hematological toxicities for patients with advanced pancreatic adenocarcinoma.

Virtual Slides: The virtual slide(s) for this article can be found here: http://www.diagnosticpathology.diagnomx.eu/ vs/13000_2014_214

Keywords: Gemcitabine, Fixed-dose rate, Pancreatic adenocarcinoma, Meta-analysis

\section{Background}

Pancreatic adenocarcinoma is one of the most aggressive solid malignancies and the eighth most common cause of cancer death worldwide [1]. Due to early metastatic dissemination, the prognosis of pancreatic adenocarcinoma is extremely poor and the 5 -year survival rate is only $6 \%[2]$.

\footnotetext{
*Correspondence: laichun_lu@126.com

${ }^{\dagger}$ Equal contributors

${ }^{3}$ Department of Pharmacy, Institute of Surgery Research, Daping Hospital, Third Military Medical University, Changjiang Road 10, Chongqing 400042 , China

Full list of author information is available at the end of the article
}

The majority of pancreatic adenocarcinoma patients are diagnosed at advanced stage and when diagnosed, the tumor is often metastasized. Therefore, systemic chemotherapy is often the treatment of choice. The administration of gemcitabine is widely used due to the evidence of clinical benefit and prolonged survival over fluorouracil [3]. While the objective response rate and survival of gemcitabine chemotherapy is still disappointing. Gemcitabine is usually administered in a manner of 30 minutes intravenous infusion, while pharmacokinetic evidence suggests that fixed-dose rate infusion $\left(10 \mathrm{mg} / \mathrm{m}^{2} /\right.$ min) of gemcitabine could optimize plasma concentration 
of gemcitabine [4-6]. In 2012, Qiu et al. reported FDR gemcitabine could not improve survival or response rate over standard gemcitabine in non-small cell lung cancer [7]. For pancreatic adenocarcinoma, FDR gemcitabine and standard gemcitabine have been compared in several randomized clinical trials (RCTs) [8-10], but no meta-analysis has been performed to systematically investigate the effects of FDR gemcitabine on treatment efficacy or toxicity. Thus, this meta-analysis was designed to compare the difference between FDR gemcitabine and standard gemcitabine in the treatment efficacy and toxic effects among patients with advanced pancreatic adenocarcinoma.

\section{Methods}

\section{Searching strategy}

Electronic databases of MEDLINE (via PubMed), EMBASE (via Ovid), and Cochrane Library were searched and the latest search was performed on September 2014. The searching strategy consisted of the following medical subheadings and key words: "pancreatic neoplasms", "pancreas cancer", "pancreatic cancer", "fixed-dose rate", "fixed dose rate", "fixed-dose-rate", "fixed dose-rate", "prolonged infusion" and "prolonged constant infusion". The detailed searching strategy was shown in the Additional file 1. References of eligible trials and relative reviews were also manually searched. There was no limitation in databases searching.

\section{Inclusion criteria}

Trials met the following criteria were included: (a) randomized controlled trials; (b) patients were pathologically confirmed of pancreatic carcinoma at advanced stage; (c) comparing FDR infusion $(10 \mathrm{mg} / \mathrm{m} 2 / \mathrm{min})$ and standard infusion (30 $\mathrm{min}$ ) of gemcitabine; and (d) published with full-text articles. Two investigators (Jiqing Xie and Jing Yuan) selected eligible trials met above criteria independently. Disagreement between two investigators was solved by discussion until consensus was achieved.

\section{Data abstraction}

Two investigators (Jiqing Xie and Jing Yuan) extracted data from eligible trials independently with a pre-designed data extraction form. And disagreement was discussed with another expert (Laichun $\mathrm{Lu}$ ) until consensus was achieved. The following data were abstracted from each eligible clinical trial: first author, publishing time, country, chemotherapy regimens, number of patients, age, number of male, number of patients with metastatic pancreatic carcinoma, number of patients with complete response and partial response, 1-year survival rate, median survival, time to treatment failure, grade $3 / 4$ hematological and non-hematological toxicities. Tumor response was measured according to Response Evaluation Criteria in Solid Tumors (RECIST). National Cancer Institute Common
Toxicity Criteria version 3.0 was used for toxicity reporting. The research protocol was in strict compliance with the institutional guidelines of the Ethics Committee at the Third Military Medical University.

\section{Validity assessment}

Methodology quality of included trials was also assessed using the "risk of bias" tool recommended by Cochrane Handbook V5.0.2. Sequence generation, allocation concealment, blinding, incomplete data and selective reporting were assessed. Based on the methods reported in each trial, each of above five components was graded "yes", "unknown" or "no", which meant low risk of bias, uncertain of bias and low risk of bias respectively.

\section{Statistical analysis}

The primary endpoints were treatment efficacy (overall response rate, 1-year survival rate, median survival, and time to treatment failure) and toxicities were secondary endpoints (neutropenia, thrombocytopenia, anemia, and vomiting). Mean differences were calculated for median survival and time to treatment failure. Relative risks (RRs) were calculated for the other endpoints. 95\% confidence intervals $(95 \% \mathrm{CI})$ were also calculated for all endpoints. A RR with a 95\% CI without 1 and the mean difference with a 95\% CI without 0 is considered of statistical significance. Fixedeffects model was used for calculating RRs and median differences unless heterogeneity was significant across trials [11]. A RR $>1$ indicates that FDR infusion is associated with a higher incidence of response, 1-year survival rate and lower incidence of grade 3/4 toxicities; and a mean difference $>0$ suggests that the FDR infusion of gemcitabine increases the median survival and time to treatment failure. Heterogeneity was tested using the Q-statistic and $\mathrm{I}^{2}$. A P value less than 0.1 or $\mathrm{I}^{2}>50 \%$ indicated that there existed significant heterogeneity [11]. A funnel plot was used to test publication bias [12,13]. All statistical analyses were carried out by Review Manager 5 (Version 5.2, The Cochrane Collaboration).

\section{Results}

After comprehensive database searching, 216 records were retrieved. By screening titles and abstracts and further reviewing of full-text papers, 3 eligible RCTs were included and analyzed in this meta-analysis. The process of study selection was shown in Figure 1. The baseline characteristics of eligible trials were shown in Table 1 . 764 patients with confirmed pancreatic adenocarcinoma contributed to the pooled meta-analysis. Most of enrolled patients had metastatic pancreatic carcinoma.

\section{Primary endpoints}

The primary object of this meta-analysis was to compare the differences of treatment efficacy between FDR 


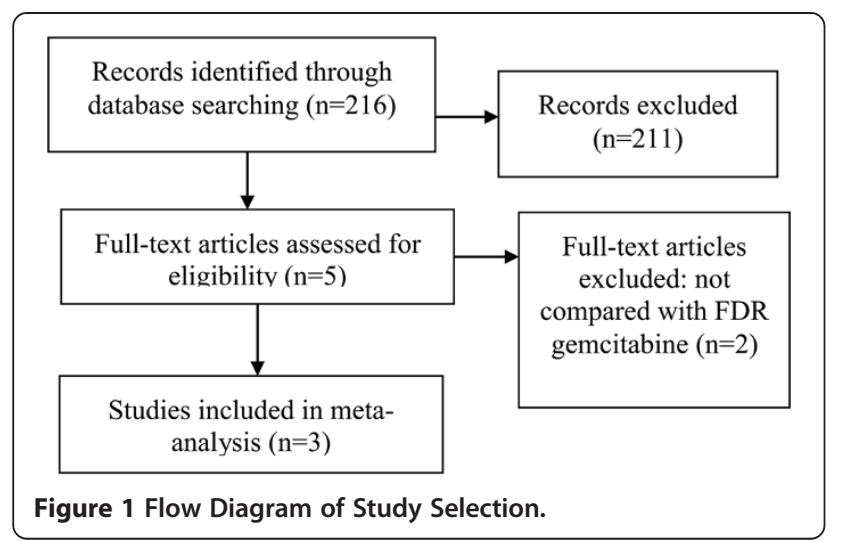

gemcitabine and standard gemcitabine. To this end, we calculated the pooled 1-year survival rate, median survival, and overall response rate. As shown in Table 2, FDR gemcitabine provided a significantly long median survival (Mean Difference $=1.24$ months, 95\% CI: 0.392.09 months; Figure 2) than standard gemcitabine, while there was no significant difference in overall response rate, 1-year survival rate, or time to treatment failure. As shown, no significant heterogeneity between studies were detected. Visual assessment of funnel plot did not reveal any evidence of publication bias (Figures not shown).

\section{Secondary endpoints}

Grade 3/4 toxic events were the secondary endpoints of this meta-analysis. Neutropenia, thrombocytopenia, anemia and vomiting were analyzed. As shown in Table 2, compared with standard gemcitabine, FDR gemcitabine significantly increased the incidence of hematologic toxicities (neutropenia, thrombocytopenia, and anemia; compassion of neutropenia was shown in Figure 3), while there was no difference in the incidence of vomiting. As shown in Table 2, no evidence of heterogeneity was
Table 2 Meta-analysis results

\begin{tabular}{|c|c|c|}
\hline Outcome & $\mathbf{R R}^{\mathrm{a}}$ & Heterogeneity \\
\hline \multicolumn{3}{|l|}{ Primary Endpoints } \\
\hline Overall response rate & $1.65[0.99,2.75]$ & $P=0.45,12=0 \%$ \\
\hline 1-year survival rate & $1.35[0.98,1.86]$ & $P=0.12,12=59 \%$ \\
\hline Median Survival & $1.24[0.39,2.09]^{b}$ & $P=0.42,12=0 \%$ \\
\hline Time to Treatment Failure & $0.54[0.25,1.33]^{b}$ & $P=0.22,12=33 \%$ \\
\hline \multicolumn{3}{|l|}{ Secondary Endpoints } \\
\hline Neutropenia & $1.79[1.49,2.15]$ & $P=0.92,12=0 \%$ \\
\hline Thrombocytopenia & $2.19[1.64,2.93]$ & $P=0.50,12=0 \%$ \\
\hline Anemia & $1.68[1.15,2.45]$ & $P=0.45,12=0 \%$ \\
\hline Vomiting & $1.31[0.89,1.93]$ & $P=0.79,12=0 \%$ \\
\hline
\end{tabular}

found. Funnel plots also showed no publication bias (Figures not shown).

\section{Discussion}

In this meta-analysis, we found FDR gemcitabine, compared with standard gemcitabine, could provide longer median survival for patients with advanced pancreatic carcinoma, and patients received FDR gemcitabine would experience more hematological toxicities.

Burris HA and colleagues reported in 1997 that singleagent gemcitabine administered as weekly 30 -min infusion could offer significantly longer survival and clinical benefit than 5-fluorouracil [3]. After that gemcitabine has been the standard chemotherapy for advancer pancreatic carcinoma. Gemcitabine (2',2'-difluorodeoxycytidine) is a prodrug and potent cytotoxic agent. The deoxycytidine kinase phosphorylate gemcitabine to gemcitabine monophosphate, and subsequent phosphorylation steps yielded gemcitabine diphosphate and gemcitabine triphosphate, which are active metabolites of gemcitabine $[4,14]$. The gemcitabine

Table 1 Baseline characteristics of eligible trials

\begin{tabular}{|c|c|c|c|c|c|c|c|}
\hline Author & Year & Country & Num $^{a}$ & Male & $\mathrm{Age}^{\mathrm{b}}$ & Metastasis & Chemotherapy \\
\hline \multirow[t]{2}{*}{ Kulke MH } & 2009 & USA & 58 & 38 & $58.9(31-81)$ & 58 & $\begin{array}{l}\text { gemcitabine } 1500 \mathrm{mg} / \mathrm{m}^{2} \text { infusion } 10 \mathrm{mg} / \mathrm{m}^{2} / \mathrm{min} \\
\text { on days } 1,8 \text {, and } 15 \text {, every } 28 \text { days }\end{array}$ \\
\hline & & & 62 & 35 & $58.9(36-84)$ & 62 & $\begin{array}{l}\text { gemcitabine } 1000 \mathrm{mg} / \mathrm{m}^{2} 30 \text {-minute infusion } \mathrm{d} 1,8 \text {, } \\
\text { and } 15 ; \text { Cisplatin } 50 \mathrm{mg} / \mathrm{m}^{2} 30 \text {-minutes infusion on } \\
\text { days } 1 \text { and } 15 \text {, every } 28 \text { days }\end{array}$ \\
\hline \multirow[t]{2}{*}{ Poplin E } & 2009 & USA & 277 & 160 & $61(36-87)$ & 246 & $\begin{array}{l}\text { gemcitabine } 1500 \mathrm{mg} / \mathrm{m}^{2} \text { infusion at } 10 \\
\mathrm{mg} / \mathrm{m}^{2} / \mathrm{min} \text { days } 1,8 \text {, and } 15 \text { every } 28 \text { days cycle }\end{array}$ \\
\hline & & & 275 & 155 & 64(31-88) & 248 & $\begin{array}{l}\text { gemcitabine } 1000 \mathrm{mg} / \mathrm{m}^{2} / 30 \mathrm{~min} \text { weekly for } 7 \text { week, } \\
\text { rest } 1 \text { week, then } 1000 \mathrm{mg} / \mathrm{m}^{2} / 30 \text { min weekly for } 3 \\
\text { week and rest } 1 \text { week }\end{array}$ \\
\hline \multirow[t]{2}{*}{ Tempero $\mathbf{M}$} & 2003 & USA & 43 & 27 & $62(42-85)$ & 38 & $\begin{array}{l}\text { gemcitabine } 1500 \mathrm{mg} / \mathrm{m}^{2} \text { infusion at a rate of } 10 \\
\mathrm{mg} / \mathrm{m}^{2} / \mathrm{min}, \mathrm{d} 1,8 \text {, and } 15\end{array}$ \\
\hline & & & 49 & 32 & $62(31-89)$ & 46 & $\begin{array}{l}\text { gemcitabine } 2200 \mathrm{mg} / \mathrm{m}^{2} \text { infusion over } 30 \mathrm{~min} \text {, } \\
\mathrm{d} 1,8 \text {, and } 15\end{array}$ \\
\hline
\end{tabular}




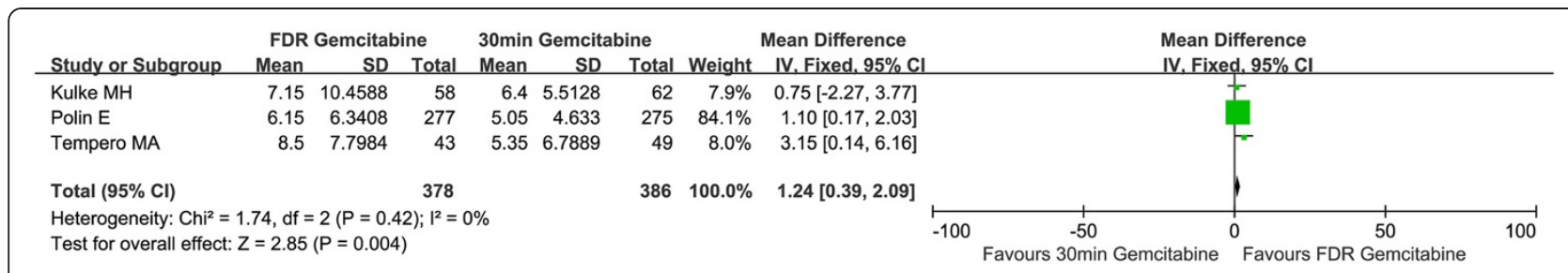

Figure 2 Forest plot for the comparison of median survival. FDR gemcitabine provided longer median survival than standard gemcitabine (Mean Difference $=1.24$ months, 95\% Cl: 0.39-2.09). FDR: fixed-dose rate; SD: standard deviation; Cl: confidence intervals.

triphosphate could inhibited cell proliferation and induce apoptosis by incorporation into DNA $[15,16]$. The deoxycytidine kinase is the rate-limiting enzyme in the accumulation of the active diphosphate and triphosphate metabolites [4]. Grunewald et al. and Abbruzzese et al. demonstrated that the dose rate about $10 \mathrm{mg} / \mathrm{m} 2 / \mathrm{min}$ could maximize the rate of formation of gemcitabine triphosphate for mononuclear cells $[4,6]$. In addition to these pharmacokinetic studies and preclinical studies, Tempero et al. reported a randomized phase II trial in 2003 and found FDR gemcitabine significantly increased 1-year and 2-year survival rates compared with 30-min gemcitabine [10]. In 2009, Poplin and colleagues showed that FDR gemcitabine could not provide substantially improved survival or symptom benefit over 30-min gemcitabine [9]. Thus, we conducted this meta-analysis to compare the treatment efficacy and toxicity between FDR gemcitabine and 30-min gemcitabine in patients with advanced pancreatic adenocarcinoma.

In this meta-analysis, we found FDR gemcitabine could only increase median survival of patients with pancreatic adenocarcinoma over standard gemcitabine, and there was no difference in overall response rate, 1-year survival rate, or time to treatment failure. This could be explained by the following reasons. First, the sample size or the number of eligible trials of this meta-analysis was small. As shown in Table 2, the results of overall response rate $(R R=1.65$, 95\% CI: 0.99-2.75) and 1-year survival rate ( $R R=1.35,95 \%$ CI: 0.98-1.86) were marginally significant, and it was highly possible that the results of response and 1-year survival would be statistically significant when further trials are reported. Second, most patients enrolled in this meta- analysis had metastatic disease (Table 1) and the prognosis of metastatic pancreatic cancer is very poor. This could explain why the median survival was significantly longer for patients received FDR gemcitabine but the 1-year survival rate was not significantly different. Because the prognosis for metastatistic patients was extremely poor and most patients died with one year. For toxicities, patients with FDR gemcitabine experienced substantially higher incidence of hematologic toxicities (neutropenia, thrombocytopenia, and anemia) than those with 30-min gemcitabine, but there was no difference in the incidence of vomiting. Compared with 30-min gemcitabine, the infusion time was prolonged for FDR gemcitabine (120 min vs. $30 \mathrm{~min}$ ), and the exposure time for blood cells was longer. Thus, the results for hematologic toxicities were expectable. Additionally, it has also been demonstrated that FDR gemcitabine could increase the incidence of hematologic toxicities in nonsmall cell lung cancer by Qiu et al. [7].

This is the first meta-analysis comparing treatment efficacy and toxicities between FDR gemcitabine and standard 30-min gemcitabine in pancreatic adenocarcinoma. No significant heterogeneity was found and funnel plots revealed no evidence of publication bias. Additionally, the evidence level of this meta-analysis was evaluated by the GRADE profiler, which facilitates the clinical application of our results. On the other hand, the major limitation of this meta-analysis lies in that the sample size of this meta-analysis was small. To include all potential trials, we designed comprehensive searching strategy and searched the 3 comprehensive databases. In spite of the effort to perform a comprehensive meta-analysis, only 3 eligible trials

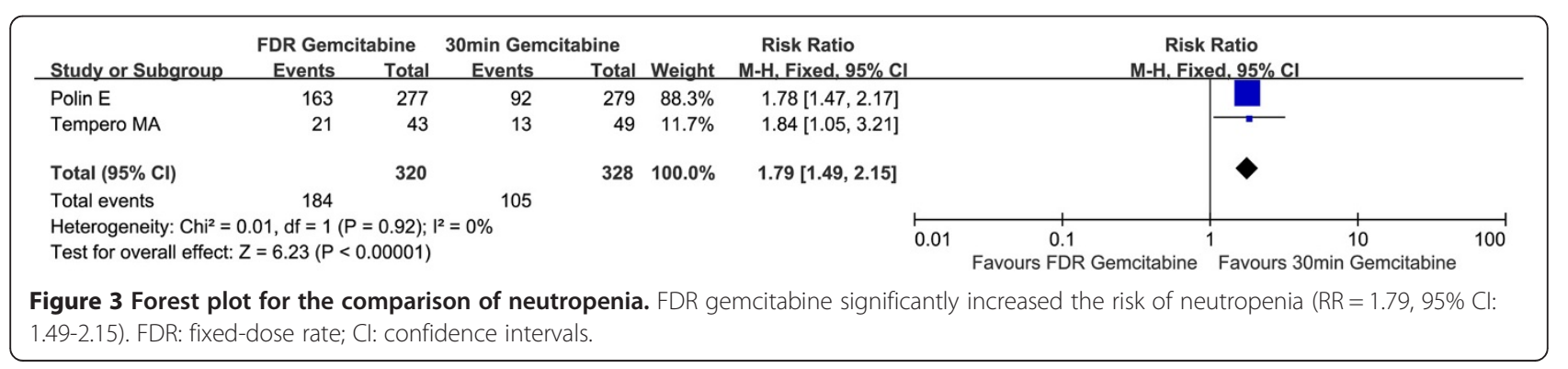


were included. Further studies are warranted to validate our findings.

\section{Conclusions}

In conclusion, our meta-analysis demonstrated that compared with standard 30-min gemcitabine, FDR gemcitabine provided longer median survival but there was no significant difference in overall response rate and 1-year survival rate in patients with advanced pancreatic adenocarcinoma, while FDR infusion was associated with more grade $3 / 4$ hematological toxicities.

\section{Additional file}

Additional file 1: Searching Strategy of this meta-analysis.

\section{Competing interests}

The authors declare that they have no competing interest.

\section{Authors' contributions}

$X J, Y J$, and $L L$ conceived, designed the study. $X J$ and $L L$ analyzed the data. $X J, Y J$, and LL wrote the paper. All authors read and approved the final manuscript.

\section{Author details}

${ }^{1}$ Department of Pharmacy, General Hospital of Jinan Military Command, Jinan 250031, China. '2Department of Medical Information, General Hospital of Jinan Military Command, Jinan 250031, China. ${ }^{3}$ Department of Pharmacy, Institute of Surgery Research, Daping Hospital, Third Military Medical University, Changjiang Road 10, Chongqing 400042, China.

Received: 6 September 2014 Accepted: 26 October 2014

Published online: 25 November 2014

\section{References}

1. Parkin DM, Bray F, Ferlay J, Pisani P: Global cancer statistics, 2002. CA Cancer J Clin 2005, 55:74-108.

2. Kris MG, Benowitz SI, Adams S, Diller L, Ganz P, Kahlenberg MS, Le QT, Markman M, Masters GA, Newman L, Obel JC, Seidman AD, Smith SM, Vogelzang N, Petrelli NJ: Clinical cancer advances 2010: annual report on progress against cancer from the American Society of Clinical Oncology. J Clin Oncol 2010, 28:5327-5347.

3. Burris HA 3rd, Moore MJ, Andersen J, Green MR, Rothenberg ML, Modiano MR, Cripps MC, Portenoy RK, Storniolo AM, Tarassoff P, Nelson R, Dorr FA Stephens CD, Von Hoff DD: Improvements in survival and clinical benefit with gemcitabine as first-line therapy for patients with advanced pancreas cancer: a randomized trial. J Clin Oncol 1997, 15:2403-2413.

4. Grunewald R, Kantarjian H, Keating MJ, Abbruzzese J, Tarassoff P, Plunkett W: Pharmacologically directed design of the dose rate and schedule of 2',2'-difluorodeoxycytidine (Gemcitabine) administration in leukemia. Cancer Res 1990, 50:6823-6826.

5. Hughes TL, Hahn TM, Reynolds KK, Shewach DS: Kinetic analysis of human deoxycytidine kinase with the true phosphate donor uridine triphosphate. Biochemistry 1997, 36:7540-7547.

6. Abbruzzese JL, Grunewald R, Weeks EA, Gravel D, Adams T, Nowak B, Mineishi S, Tarassoff P, Satterlee W, Raber MN, Raber MN: A phase I clinical, plasma, and cellular pharmacology study of gemcitabine. J Clin Oncol 1991, 9:491-498.

7. Qiu MT, Ding XX, Hu JW, Tian HY, Yin R, Xu L: Fixed-dose rate infusion and standard rate infusion of gemcitabine in patients with advanced non-small-cell lung cancer: a meta-analysis of six trials. Cancer Chemother Pharmacol 2012, 70:861-873.

8. Kulke MH, Tempero MA, Niedzwiecki D, Hollis DR, Kindler HL, Cusnir M, Enzinger PC, Gorsch SM, Goldberg RM, Mayer RJ: Randomized phase II study of gemcitabine administered at a fixed dose rate or in combination with cisplatin, docetaxel, or irinotecan in patients with metastatic pancreatic cancer: CALGB 89904. J Clin Oncol 2009, 27:5506-5512.

9. Poplin E, Feng $Y$, Berlin J, Rothenberg ML, Hochster H, Mitchell E, Alberts $S$, O'Dwyer P, Haller D, Catalano P, Cella D, Benson AB: Phase III, randomized study of gemcitabine and oxaliplatin versus gemcitabine (fixed-dose rate infusion) compared with gemcitabine (30-minute infusion) in patients with pancreatic carcinoma E6201: a trial of the Eastern Cooperative Oncology Group. J Clin Oncol 2009, 27:3778-3785.

10. Tempero M, Plunkett W, Ruiz Van Haperen V, Hainsworth J, Hochster H, Lenzi R, Abbruzzese J: Randomized phase II comparison of dose-intense gemcitabine: thirty-minute infusion and fixed dose rate infusion in patients with pancreatic adenocarcinoma. J Clin Oncol 2003, 21:3402-3408

11. Lau J, loannidis JP, Schmid CH: Quantitative synthesis in systematic reviews. Ann Intern Med 1997, 127:820-826.

12. Egger M, Davey Smith G, Schneider M, Minder C: Bias in meta-analysis detected by a simple, graphical test. BMJ 1997, 315:629-634.

13. Sterne JA, Egger M: Funnel plots for detecting bias in meta-analysis: guidelines on choice of axis. J Clin Epidemiol 2001, 54:1046-1055.

14. Heinemann V, Hertel LW, Grindey GB, Plunkett W: Comparison of the cellular pharmacokinetics and toxicity of 2',2'-difluorodeoxycytidine and 1-beta-D-arabinofuranosylcytosine. Cancer Res 1988, 48:4024-4031.

15. Huang P, Chubb S, Hertel LW, Grindey GB, Plunkett W: Action of 2',2'difluorodeoxycytidine on DNA synthesis. Cancer Res 1991, 51:6110-6117.

16. Huang P, Plunkett W: Fludarabine- and gemcitabine-induced apoptosis: incorporation of analogs into DNA is a critical event. Cancer Chemother Pharmacol 1995, 36:181-188.

doi:10.1186/s13000-014-0214-8

Cite this article as: Xie et al:: Gemcitabine fixed-dose rate infusion for the treatment of pancreatic carcinoma: a meta-analysis of randomized controlled trials. Diagnostic Pathology 2014 9:214.

\section{Submit your next manuscript to BioMed Central and take full advantage of:}

- Convenient online submission

- Thorough peer review

- No space constraints or color figure charges

- Immediate publication on acceptance

- Inclusion in PubMed, CAS, Scopus and Google Scholar

- Research which is freely available for redistribution 